\title{
NETWORKING LAYER FOR THE EVOLUTION OF MARITIME PORTS INTO A SMART ENVIRONMENT
}

\author{
A. Haidine ${ }^{(1)}$, A. Ait-Allal ${ }^{(2)}$, A. Aqqal ${ }^{(3)}$ and A. Dahbi ${ }^{(4)}$ \\ ${ }^{(1)}$ Dept. TRI, National School of Applied Sciences, El Jadida, Morocco - haidine.a@ucd.ac.ma \\ ${ }^{(2)}$ Lab SSDIA , Ecole Normale Supérieure de l'Enseignement Technique, Mohammedia, Morocco -aitallal.abdelmoula@gmail.com \\ ${ }^{(3)}$ Dept. TRI, National School of Applied Sciences, El Jadida, Morocco - aqqal.a@ucd.ac.ma \\ ${ }^{(4)}$ Dept. TRI, National School of Applied Sciences, El Jadida, Morocco - dahbi.a@ucd.ac.ma
}

KEY WORDS: Smart port, IoT architecture, terminal operation system, 4G/5G, smart grid, WSN, LPWA.

\begin{abstract}
:
The maritime transport is playing an increasing and critical role on the worldwide trade; because it guarantee the delivery of $80 \%$ of the goods worldwide. The maritime ports are the articulation of the maritime transports, and therefore are responsible of (long delay in the ports means high risks for goods deterioration, higher costs for the logistics companies, longer occupation of containers for containers management companies, etc. In recent years some publications are talking about smart ports. In this paper we take some of the most recent models of smart port, and propose some extensions by considering the recent recommendations from International Maritime Organization (IMO), which have been translated and issued legislation frameworks by the European Union, such as maritime environment monitoring, air pollution, etc. In the recent years, any "smart" environment is modeled through an Internet-ofThings (IoT) layered model. Thus, we discuss the model of smart port environment as IoT layered model, where the networking/communications layer plays the core role. Furthermore, we analyse the possible networking technologies which could support the different smart domains building the smart port environment. This is achieved by first defining the different sub-layers of the hierarchical communication layer, and then comparing different technologies for building the broadband mobile sub-layer.
\end{abstract}

\section{INCTRODUCTION}

In international trade, no transport sector is as critical as the maritime sector. Roughly $80 \%$ of all goods are transported by sea, and in terms of tonnes per kilometre travelled, shipping is the most efficient and cost-effective transport mode, [Bodewig20]. In the example of Europe, the maritime transport sector also forms a big part of the intra-European transport system, facilitating and redistributing trade flows from landbased route networks, while contributing to the efforts to reduce the overall external environmental and social costs from transport.

The maritime ports are the articulation of the maritime transports, and therefore are responsible of (long delay in the ports means high risks for goods deterioration, higher costs for the logistics companies, longer occupation of containers for containers management companies, etc. In the recent years some publications are talking about smart ports. In this paper we take some of the most recent models of smart port, and proposes some extensions by considering the recent recommendations from International Maritime Organization (IMO), which have been translated and issued legislation frameworks in/by the European Union. Usually, any "smart" environment is modelled through an Internet-of-Things (IoT) layered model. Thus, we discuss the model of smart port environment as IoT layered model, where the networking/communications layer plays the core role.

Furthermore, we analyse the possible networking technologies which could support the different "smart" applications from different smart domains to the smart port environment. This is achieved by first defining the different sub-levels of the hierarchical communication layer. For the evaluation of the optimal solution for the broadband mobile sub-layer, we consider 2G/2.5G (GSM/GPRS), 3G/3G+ (UMTS/HSPA+), 4G/4.5G (LTE-A/LTE-A Pro), WiMAX (as previous 4G candidate) and $5 \mathrm{G}$. A qualitative comparison of some main performance criteria is discussed. It is clear that GSM/GPRS is not a broadband solution, but we cited here because different literature are considering it as optimal solution for their own application, like connecting the sink-node of WSN to their server, or in smart metering, or smart street lighting, etc. But, an optimal solution should be able to support as much as possible of applications from vertical smart domains (from smart port environment, which is our focus).

The remaining of the paper is organized as follows: in the second section, we discusses the different definitions and models of smart ports from recent literature and then we propose some extensions based on some International maritime Organization (IMO) and EU directives, followed by IoT layered model, which describes any smart environment. The third section describes the main smart domains that govern some of the laments in the smart port environment, such as smart grid and micro-grids. The fourth section describes the three sublayers building the communications/networking layer of the smart port IoT architecture, followed by a comparison of candidate solutions, including 5G. Finally, we give conclusions and some perspectives for future work.

\section{NEEDS AND REQUIRMENTS FOR SMART PORTS}

\subsection{Characteristics of Smart Port}

According to [1], the maritime ports have evolved over five generations. The 1 st generation served as a nodal point of land and sea transports and provided basic operations, such as logistics and transportation, cruise, fishing, and emergency 
rescue. Then the 2 nd generation deployed equipment and infrastructures to reduce the dependency on manpower. The $3 \mathrm{rd}$ generation, which serves as a cargo handling center, provides value-added services such as warehousing, packaging, and distribution. The 4th generation connects physically-separated ports to serve as a networked port. Finally, the more recent and 5 th generation of ports is a customer- and community-centric smart port that is distinguished by five main features:

(a) smart port services and applications such as vessel and container management;

(b) technologies such as data center, networking and communication, and automation;

(c) use of sustainable technology to increase energy efficiency and reduce greenhouse gases emission;

(d) cluster management such as a shipping cluster that consists of geographically proximate companies and stakeholders with their main activity being shipping; and

(e) development of hub infrastructures to foster collaboration among different ports.

In this paper, we focus on technical oriented aspects of smart ports (i.e. (a), (b) and (c)), while political and strategic aspect are out of paper's scope. The authors proposed five smart applications as components for the realization of smart ports: (1) Smart vessel management, (2) Smart container management, (3) Smart port management, (4) Smart energy management and (5) Smart resource management.

In this paper, we propose to extend these smart applications by taking into consideration the recommendation and directives from the European Union for the future maritime traffic management, where the ports play an essential role. According to the EU directives, "Sustainability and Climate Change" is one of the highest priorities in the future maritime sector, which aims to the decarbonisation, air emissions and water quality \& waste management; [2]. In addition, the following topic areas all include some aspects of water and waste management, and thus they also contribute as trends impacting the maritime coastal environments:

- integrated governance and protection of the oceans

- ballast management system \& ballast water discharge

- prohibition of discharging untreated sewage in the sea

- invasive alien species (IAS).

In order to touch these EU recommendations", we propose to extend the set of smart applications that build the smart port environment, as illustrated in Figure 1. For example, we propose to add the "environment monitoring", "Smart waste management", "Smart grid" and its important component the "micro-grid", etc. Because of lack of space, we will choose some key smart applications and give details on them and their adequate communications and networking technologies, because this is the focus of our paper.

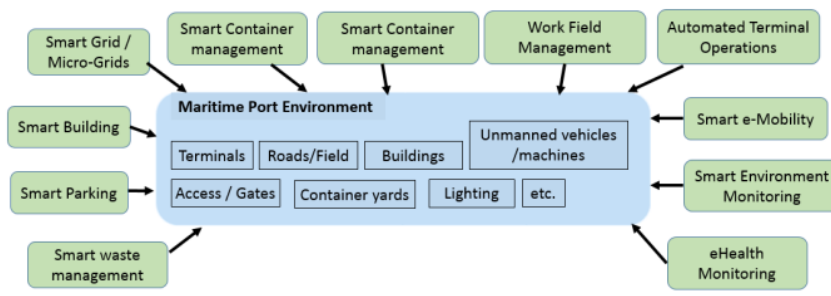

Figure 1. Extended Smart domains to make from the maritime port a "smart" environment.

\subsection{IoT Architecture Model for any "Smart" Environment}

In the recent years, a growing number of physical objects are being connected to the Internet at an unprecedented rate realizing the idea of the Internet-of-Things (IoT). There is a large number of applications that use IoT paradigm, including domains of transportation, healthcare, industrial automation, building, lighting, etc. So, we can say that any "Smart" domain or application should have an IoT architecture as basis. There are some different models used to model the IoT architecture adopted in any smart domain or application, as reviewed with details by [3]. From the pool of proposed models, they described the basic model of a three layer architecture consisting of the Application, Network, and Perception Layers. In the recent literature, however, some other models have been proposed that add more abstraction to the IoT architecture. A five layers models have further developed in some sub-variants, like the middleware-based model, the SOA-based model or just the basic five-layer model.

In this work, we consider the model illustrated in Figure 2, which will help us to describe the different layers needed to build in the maritime port as a smart environment. Furthermore, we will see that the smart port is a set of smart domains and smart applications adaptable and applicable in the port environment. For example, smart grid is a large smart domain with a number of smart applications along the whole chain generation/transmission/distribution/consumer. The smart grid applications at the consumer side (or more precisely at proconsumer side) build an important puzzle piece in the smart port environment.

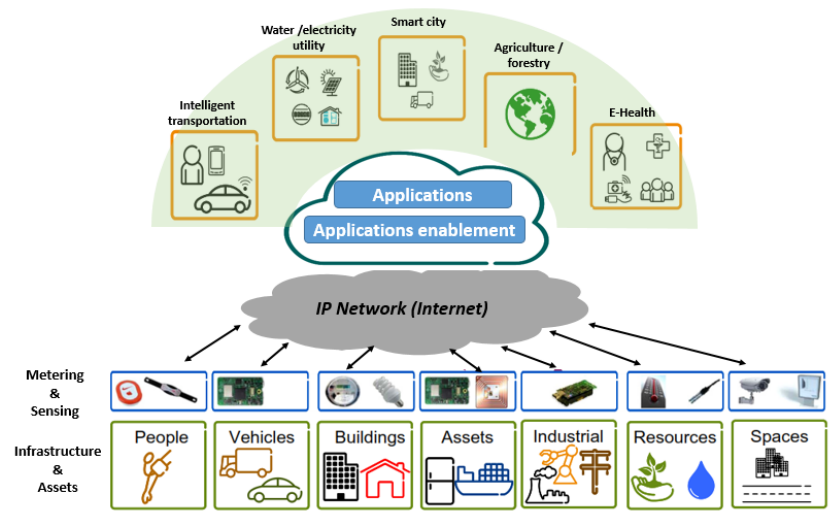

Figure 2. Network layer as core layer in different vertical smart domains.

Our work focus on the investigation of the networking layer (which sometimes simply called communications) layer, which can be divided in 4 sub-layers or levels depending on the requirements level that is dominated by the coverage and network performance metrics (i.e. throughput, delay, reliability, packet loss rate).

\section{SMART DOMAINS FOR BUILDING A SMART PORT ENVIRONMENT}

\subsection{Smart Grid and Micro-grid in Port Environment}

Smart grid in the paradigm of building a modern electricity grid where information and electricity are bi-directionally exchanged. The goal of smart grid is to build an efficient, reliable, secure and environment friendly electricity infrastructure. Addressing these needs requires that new design approaches build intrinsically on enabling technologies, including power electronics, communication, distributed decision making, distributed generation (incl. renewable sources), and integration of local energy storage in stationary and mobile platforms [7]. 
One of the major aspects of the smart grid is to be consumercentric. Different applications should be implemented to build the smart grid concept, such as ([8]):

- advanced distribution automation (DA)

- distribution feeder circuit automation .....

- advanced metering infrastructure (AMI)

- charging electric vehicles (EV) and e-Mobility

- local controllers in buildings, on microgrids, and on distribution systems for local area networks

- distributed energy resources (DER), including distributed generators, renewables, and energy storage facilities that are equipped with smart inverters and other advanced controllers. This covers also the notion of micro-grid (called also nano-grid like in [7]

- home energy management (HEM), etc.

In this work, we focus only on the smart grid applications at the consumers side and which are applicable in the context of maritime port environments: micro-grid, smart lighting, and smart metering,.

3.1.1 Micro-grid: Microgrids are intentional islands with distributed energy resources (DERs) and associated loads formed at a facility or in an electrical distribution system. Microgrids provide reliable energy, which can control the power flow when they are connected to the grid or operate autonomously in an electrical island, or in transition between these two states. A typical structure of a smart grid is illustrated in Figure 3 [9], where micro-grid is connected to the main smart grid through a point of coupling (PoC) and Connection interface (CI) (not shown on figure for simplification). The microgrid is built photovoltaic panels, wind turbine (small and medium size), power storage elements, loads and power electronic interfaces to interconnect and control them.

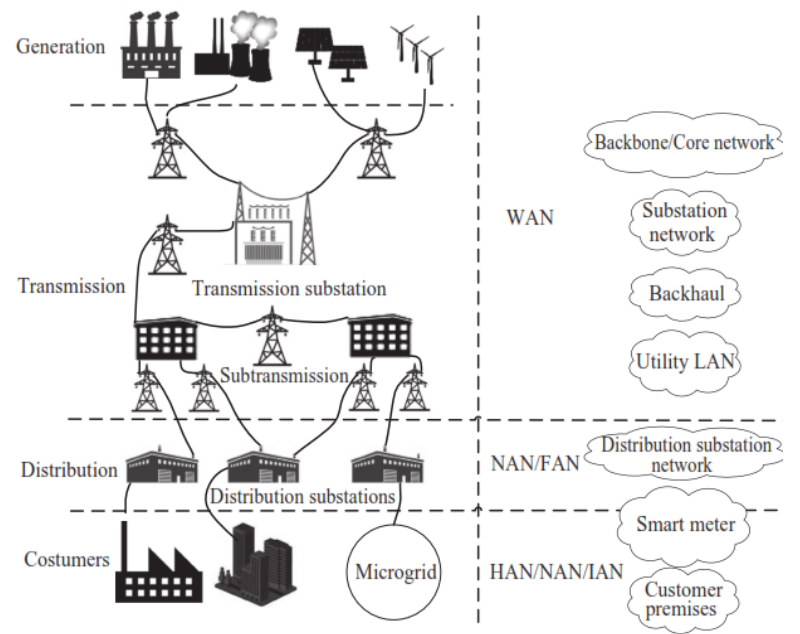

Figure 3. Positioning of micro-grid at consumer side in smart grid with different levels of networking [9].

Generally, any power utility has several micro-grids connected to its main smart grid. Therefore, a continuous communications gas to be guaranteed inside in the smart-grids to make possible the control and the management. The communications with and within the smart-grids can be modelled with a three-level model as illustrated in Figure 3 [9], where the main controller controls a number of microgrids, and the smaller microgrids regulate local operations within the local grid. Different communications technologies can be used, according to the transmission coverage. Table 1 summarizes the widely used transmission solutions for smart-grid communications.

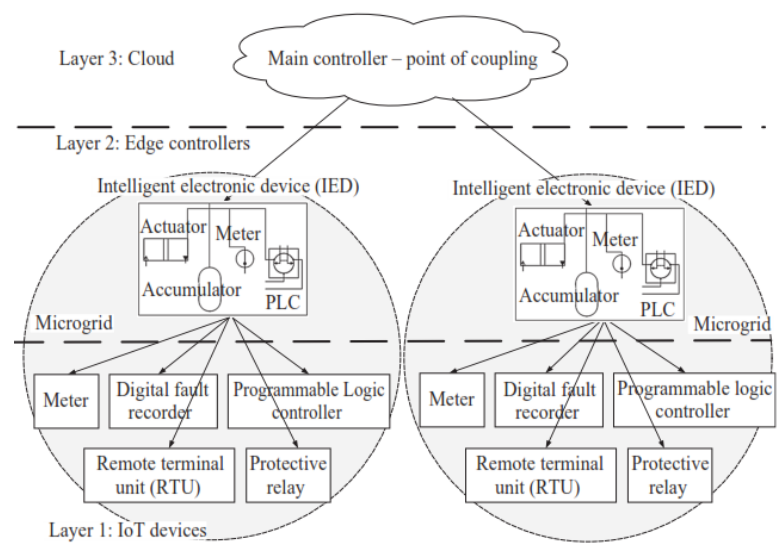

Figure 4. Communication inside of micro-grid and its connection to the control layer.

In the case of the maritime port environments, renewable energy source (i.e. micro-grids) can be in form off-shore wind energy, on-shore wind, PV panels or solar energies. Table I summarizes the most used technologies for micro-grid communications, and which should be taken into consideration for smart port environment.

\begin{tabular}{|l|l|l|}
\hline Technology & Standard & Application Area \\
\hline $\begin{array}{l}\text { Narrow } \\
\text { band PLC }\end{array}$ & $\begin{array}{l}\text { IEEE P1901.2 } \\
\text { G3-PLC } \\
\text { PRIME }\end{array}$ & HAN/NAN \\
\hline & $\begin{array}{l}\text { IEEE 1901 [21] } \\
\text { HomePlug 1.0 } \\
\text { HomePlug Turbo }\end{array}$ & \\
$\begin{array}{l}\text { Broadband } \\
\text { PLC }\end{array}$ & $\begin{array}{l}\text { HomePlug AV } \\
\text { HomePlug CC } \\
\text { HomePlug Green }\end{array}$ & HAN/BAN/NAN \\
& HomePlug BPL & \\
\hline $\begin{array}{l}\text { Wirless } \\
\text { Perosnal } \\
\text { Area } \\
\text { Network } \\
\text { (WPAN) }\end{array}$ & $\begin{array}{l}\text { IEEE 802.15 } \\
\text { (Bluetooth/ZigBee) }\end{array}$ & HAN/NAN/V2G \\
\hline Z-wave & Z-wave & HAN \\
\hline
\end{tabular}

Table 1. Communications solutions for smart grid applications that maybe adopted in smart ports (adapted from [9] [10]).

3.1.2 Smart lighting: The notion of smart lighting is a part of smart grid when applied on public area like street lighting in smart city, or part of smart building/smart campus when applied on a private and geographically very limited area. This is also applicable for the case of smart port environment. In general definition, smart lighting is a lighting technology designed for energy efficiency, convenience and security. This may include high efficiency fixtures and automated controls that make adjustments based on conditions such as occupancy of the served area or daylight availability and intensity. Lighting is the deliberate application of light to achieve some aesthetic or practical effect (e.g. illumination of a security breach). It includes task lighting depending on the work, accent lighting for special area, and general lighting with minimum requirements. A simple example for smart lighting, which seems more suitable for smart port area, is illustrated in Figure 5. 


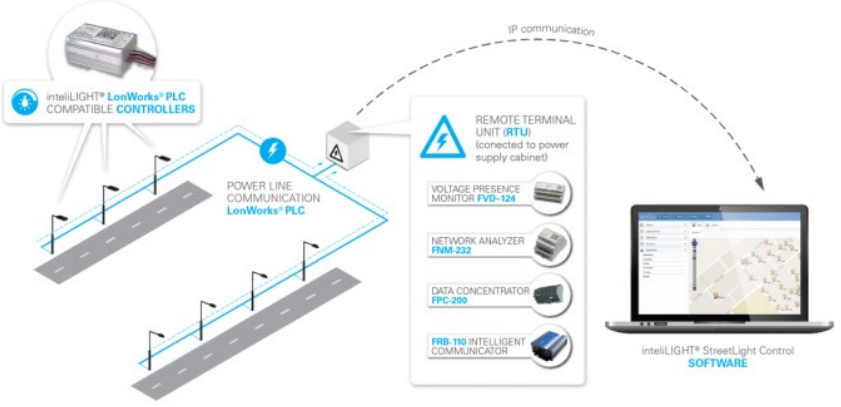

Figure 5. Smart street /field lighting using PLC-applicable also for port environments [11].

3.1.3 Special challenges for smart grid in the smart port environment: The transition of maritime port into smart environment brings additional challenges to the power supply. As example, we can cite:

- On-shore Power Supply (OPS): Vessels are consuming a large amount of electricity, even when parking in the port terminal. The electricity is generated using diesel motors, which generate huge amount of polluting dioxide gaz. Therefore, an electricity supply from the port's grid to the ships staying in terminals will reduce considerably the pollution generated by the ships in the port environment. According to [2], the environmental pillar also includes a number of local emissions which are generally of less relevance for global climate change, but very important for the health of the population in the port vicinity. This is, of course, particularly important for the so-called 'city ports' which are situated in densely populated areas.

- Charging station for e-Mobility, this is true either for autonomous vehicle (AGVs) or just electric vehicles (e-cars of employees and visitors as well as e-Trucks used in the port field for normal ports operations or e-Trucks of logistic companies).

\subsection{Smart Port Operations}

3.2.1 Automated Terminal Operation System (ATOS): According to [4], the automated terminal operations system can be modelled as an IoT system based on a sensors layer (Figure 6) connected via networking and data layer to a control and management application. Accordingly, the automatic container terminal implementation requires the use of sensing systems that are employed in tasks such as the structural health monitoring of the quayside cranes, container position detection and handling, AGV localization, navigation and control, etc. The current developments in the field of optical fibre sensors, highly sensitive magnetic sensors, and MEMS inertial measurement units that enable the interoperable wireless protocols, including the latest developments of $4 \mathrm{G}$ and $5 \mathrm{G}$ that will permit the extension of the Internet connectivity of the sensing systems, represent big opportunities for new developments in the field of smart ports. Thus, new smart sensing architectures for container identification and management, vehicle identification and management, location and navigation services, and the safety of port terminals equipment related to structural health monitoring are based on contact and remote sensing solutions.

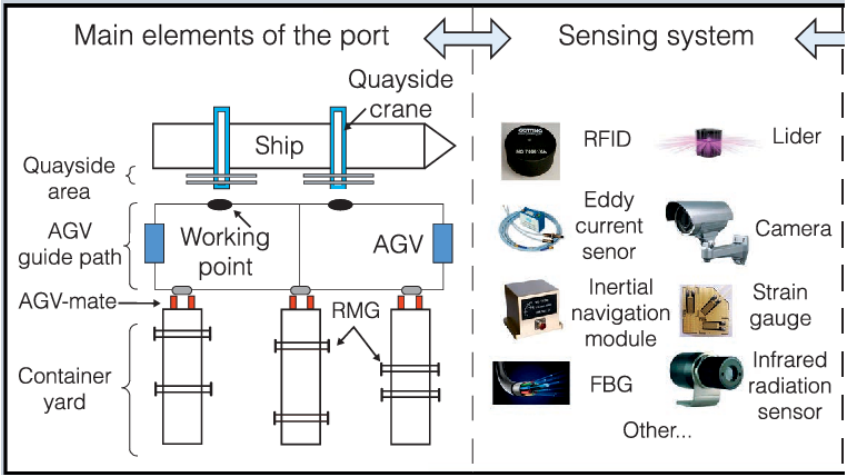

Figure 6. Assets and sensors/actuators layers building the IoT system model of ATOS [4].

3.2.2 Automated Guided Vehicles (AGV): An Automated Guided Vehicle (AGV) or Automatic Guided Vehicle is a vehicle used to transports containers (and other items) in the port's area. These vehicles are remotely controllable, but also have an advanced level of intelligence to execute self-driving and other optional decisions; as depicted in Figure 7. Several advantages are guaranteed through the use of AGVs, such as economic/cost effectiveness, time efficiency, increase the efficiency of the overall processes in the ports, considerable reduction of accidents and consequent injuries are reduced substantially because of absence of human traffic, etc.

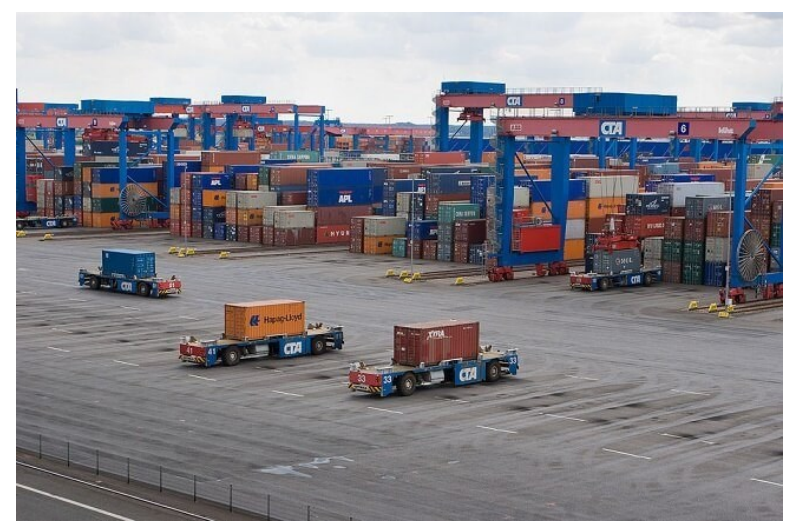

Figure 7. Fleet of AGVs as part of port operations automation (Credits: wikiwand.com).

3.2.3 UAV or Drone Assisted Operation: Drone or Unmanned Aerial Vehicles (also Unmanned Aircraft Systems UAS) are gaining a large application fields. Maritime ports are already testing drones for their operation, like [5]:

- Inspections of physical structures and patrolling of security rounds;

- Routine inspections for maintenance of buoys, pipes, docks, breakwater cranes, roof-ships and other structures that are conventionally difficult to access;

- Stock measurement to calculate (bulk) volumetric mass inventory. Inventory volume tracking of outdoor bulk material storage areas;

- Detection of irregular situations, leaks or abnormalities through (thermal and gas) sensors, as supplemental emergency support without the need to expose people to the affected areas;

- Measurement and control of environmental aspects, detection of contamination and tracking and monitoring those responsible for the environmental breaches. 
Environmental and ecological monitoring of on-site processes and surrounding areas;

\subsection{Environment Monitoring in Port- Sea-side and land- side}

Decades ago, wireless sensor networks (WSNs) have been used in control and monitoring of different environments. With the apparition of the more dominating paradigm of Internet-ofThings, WSN became an important part of IoT and its smart applications. Thus, IoT-based technologies can certainly be applied to the monitoring and protection of marine environments, as investigated in [6]. In this reference, the authors proposed that IoT-based marine environment monitoring application areas include: (1) Ocean sensing and monitoring; (2) water quality monitoring; (3) coral reef monitoring; (4) marine (either offshore or deep-sea) fish farm monitoring; (5) wave and current monitoring. Different applications use different IoT system architectures, sensing and control technologies, and communication technologies.

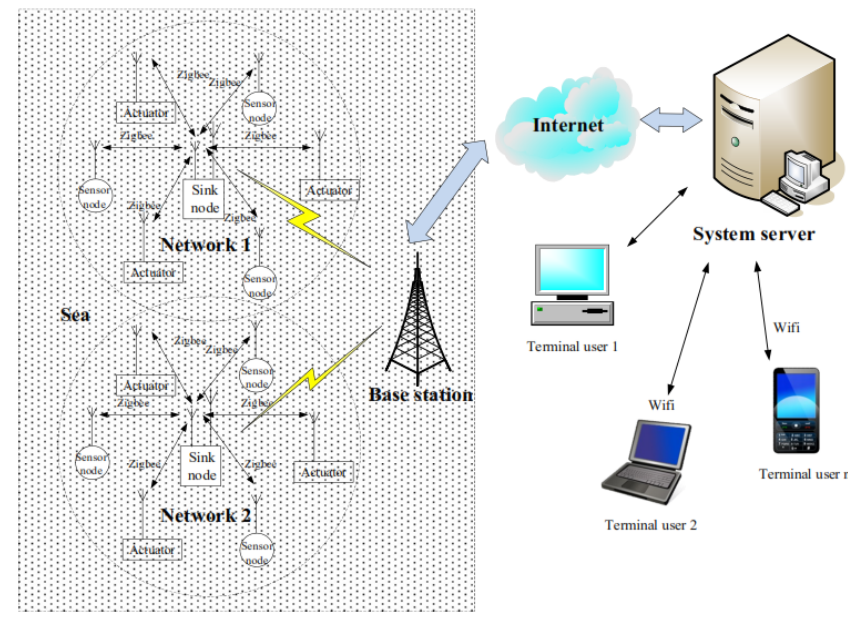

Figure 8. Networking layer for WSN in marine environment monitoring [6].

An optimal networking architecture of IoT-based marine environment monitoring and protection systems is illustrated in Figure 8. It includes sensor nodes and actuator nodes, sink nodes, a base station, a system server, and user terminals. Sensor nodes are used to sense and monitor environmental parameters such as water temperature and $\mathrm{pH}$, salinity, turbidity, oxygen density, and chlorophyll levels, and transmit the collected data to sink nodes via ZigBee or some other wireless communication protocols.

In the context of smart port environment, we propose to use (some of) these monitoring solutions to monitor the maritime environment inside the port and the neighbouring maritime zones. Furthermore, the discussed solutions of [6], did not take into consideration some optimal technologies for IoT especially 5G and LPWA represented by LoRa and SigFox solutions, as will see in the discussion of the entire networking landscape. Furthermore, this reference, and also its used literature propose the use of $2 \mathrm{G}$ (GSM/GPRS) and $3 \mathrm{G}$ (UMTS/HSPA) in the networking layer. However, mobile network operator have started switching off all $2 \mathrm{G}$ and partially $3 \mathrm{G}$ systems, because of their very bad spectral efficiency (measured in transmission capacity of bits/second/Hertz) bits/ as well as energy efficiency, compared to $4 \mathrm{G}$ (LTE/LTE-A/LTE-A-Pro).

\section{EFFICIENT NETWORKING LAYER IN SMART PORT}

\subsection{General Hierarchical Architecture in the Smart Port}

Smart port as any smart environment can be modeled by an IoT architecture. In any of the previously cited models, the networking layer represents the core layer between the sensors/actuators dispatched over the infrastructure/ assets on the field and the intelligent/decision layer of the smart applications. We have seen, that the smart port paradigm includes a set of smart domains, where each domain has its own smart applications and services.

An optimal design of the networking layer consists in having a networking infrastructure, which can support a variety of services from different vertical smart domains. A simplified networking infrastructure in a smart port environment is depicted on Figure 9. Generally, this architecture can be subdivided in three sub-layers:

- Narrow-band (NB) sub-layer: it must connect the sensors to a gateways. It should be very energy efficiency (low energy consumption) while offering low bit rates. Generally, wireless technologies like Bluetooth, Bluetooth energy (BLE), ZigBee, Z-wave, LPWA (SigFox or LoRa or WightLess) are preferred than the wired solution, as they are easy to deploy and to maintain.

- Mid-band (MB) sub-layer: this offers medium bit rates at the level of $2 \mathrm{Mbps}$, and mainly forwards the aggregated packets from the gateways in the uplink to the application layer. This could also give direct connections to end-devices with bit rates up $2 \mathrm{Mbps}$ (i.e. sensors like low-definition surveillance cameras)

- Broadband (BB) sub-layer: This is the mobile backbone of the networking layer in the smart port area. It should transport all the collected traffic from NB and MB sublayers, but also offers direct connections to end-devices with higher demand of bandwidth, like HD camera, mobile $\mathrm{PC} /$ tables/device of the field force, normal Internet services everywhere in the port area, etc.

While the NB and MB sub-layers can be considered as private networks (owned and managed locally), the broadband sublayer should use the infrastructure and services offered by the national mobile network operator(s). This part is very crucial, because this sub-layer plays a core or hub role in the smart port environment and any problems (congestion, failure, malfunctioning, etc.) will affect mostly all the smart domains. The adequate technologies are discussed in the next subsection.

\subsection{Efficient Technologies for the Broadband Mobile Core/Backhaul}

For the evaluation of the optimal solution for the broadband mobile sub-layer, we consider $2 \mathrm{G} / 2.5 \mathrm{G}$ (GSM/GPRS), 3G/3G+ (UMTS/HSPA+), 4G/4.5G (LTE-A/LTE-A Pro), WiMAX (as previous $4 \mathrm{G}$ candidate) and $5 \mathrm{G}$. A qualitative comparison of some performances are depicted in Figure 10. It is clear that GSM/GPRS is not a broadband solution, but we cited here because different literature are considering it as optimal solution for their own application, like connecting the sink-node of WSN to their server, or in smart metering, or smart street lighting, etc. But, an optimal solution should be able to support as much as possible of applications from vertical smart domains (from smart port environment, which is our focus). Furthermore, mobile operator have started switching off their $2 \mathrm{~g} / 2.5 \mathrm{G}$ systems, because of their: i) very bad spectral efficiency; ii) high costs for each transmitted Megabyte (MB) 
and iii) very bad energy efficiency (i.e. very energy consumption). This becoming also reality for $3 \mathrm{G}$ systems. WiMAX was considered in the past as a $4 \mathrm{G}$ candidate, because it offers similar performances like the first version of LTE (Release 8). But WiMAX is not developed anymore and therefore not more optimized for new and modern services/applications. Thus, we have $4 \mathrm{G} / 4.5 \mathrm{G}$ and $5 \mathrm{G}$ as real candidate for smart port.

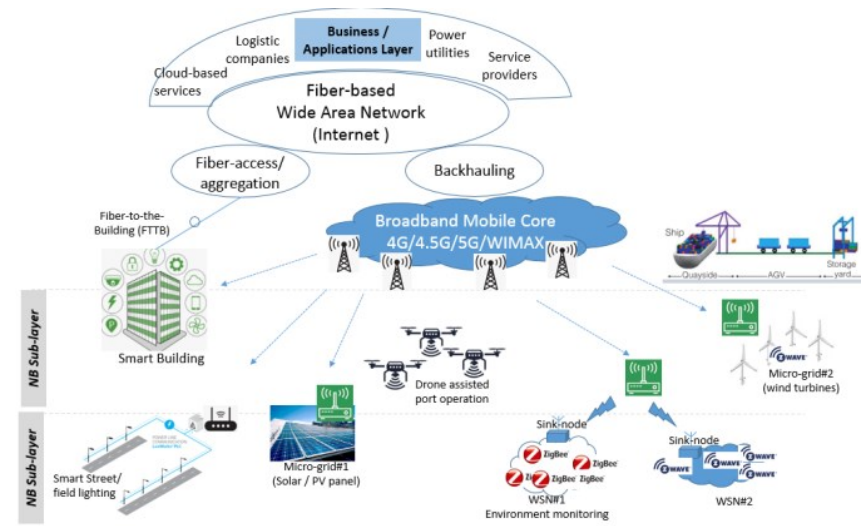

Figure 9. Sub-layers of communications architecture in smart port.

The $5 \mathrm{G}$ technology as the recent technology offers a large technical advantageous aspects, however, it suffers from a delayed roll-out for different reasons, among which we can cite:

- This was first caused by a delay while issuing the corresponding 3GPP 5G standards. Thus, the roll-out was postponed for the end of 2020.

- But, the COVID-19 pandemic crisis had a strong impact on the economy worldwide and on the strategic planning of international mobile operators.

- Furthermore, the $5 \mathrm{G}$ equipment manufacturing was led by HUAWEI (exactly like with 4G/LTE), and several international operators were expecting a strong participation of the Chinese company in their $5 \mathrm{G}$ roll-out. Because of different reasons (geo-political, trade war, etc.), HUAWEI was banned from almost all occidental telecom markets (in US, Canada and Western Europe). As result, the roll-out was once again delayed and the deployment costs will probably and considerably increase

- In addition, LTE-Advanced (4G) and LTE-Advanced Pro $(4.5 \mathrm{G})$ are already using some key technologies such massive MIMO and beamforming for extremely high throughput (> Gbps), highly flexible and adaptable bandwidth to adapt its throughput to any service or application (Narrow-band IoT LTE, Machine-to-Machine or M2M for mid-band requirements). So, for the mobile network operator there is no emergency to deploy a rollout of $5 \mathrm{G}$ at any costs

- Research and development works have started dealing with Beyond $5 \mathrm{G}(\mathrm{B} 5 \mathrm{G})$ or $6 \mathrm{G}$. Maybe, it is worth for mobile operator to analyse a migration directly to $6 \mathrm{G}$ without a full deployment of $5 \mathrm{G}$.

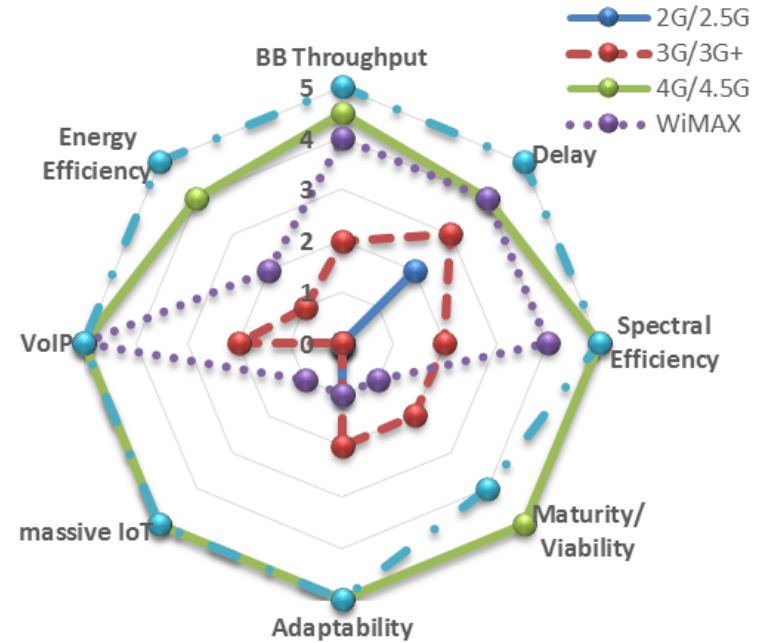

Figure 10. Comparison of technologies for BB sub-layer in smart port.

\section{CONCLUSIONS}

In this paper we discussed some existing definitions and recent models of smart maritime ports. Furthermore, we extended them by considering some import issues such as environment monitoring, on sea-side as well as land-side of the port environment, smart grid and micro-grid in the context of sea ports, etc. Finally we give the entire networking landscape, which should support different services and applications from different/vertical smart domains in the port environment. Such architecture landscape has three sub-levels, narrowband, midband and broadband. This later one is the most challenging decision. From a qualitative comparison of some main performance criteria (such as throughput, flexibility and adaptability to large set of applications/services, etc.), we believe that the 4G/4.5G (LTE-advanced / LTE-Advanced Pro) is most suitable for smart ports, even if $5 \mathrm{G}$ as the most recent generation seems to be more advantageous.

As perspective, we will build simulations of the networks on different port environment scenarios with traffic sources parameterized according to the smart applications. Such simulation will allow us a quantitative evaluation of the network performance under different scenario conditions, and also with different communications technologies.

\section{REFERENCES}

[1] K.A. Yau, S. Peng, J. Qadir, Y.C. Low and M.H. Ling: "Towards Smart Port Infrastructures: Enhancing Port Activities Using Information and Communications Technology," in Journal IEEE Access, April 2020, DOI:10.1109/ACCESS.2020.2990961.

[2] K. Bodewig: "Shaping the future policy of the European Maritime Space Motorways of the Sea Detailed Implementation Plan of the European Coordinator" Report EU Mobility and Transport, June 2020.

[3] Al-Fuqaha, A., Guizani, M., Mohammadi, M., Aledhari, M. and Ayyash M. Internet of Things: A Survey on Enabling Technologies, Protocols and Applications. IEEE Communications Surveys \& Tutorials. 17. 4th Q 2015. 10.1109/COMST.2015.2444095.

[4] Yang, Y., Zhong, M., Yao, H., Yu, F., Fu, X., \& Postolache, O. (2018). Internet of things for smart ports: Technologies and 
challenges. IEEE Instrumentation \& Measurement Magazine, $21,34-43$.

[5] V. Bexiga: "Blue Innovation - Drones in Port Operations," Escola Europa Intermodal Transport, online https:/escolaeuropea.eu/odiseo/issue-30-spring-2019/blueinnovation-drones-in-port-operations, last retrieved on 23.06.2021.

[6] G. Xu, X. Sun and W. Shen: "Internet of Things in Marine Environment Monitoring: A Review," Sensors 2019, 19, 1711; April 2019, doi: 10.3390/s19071711.

[7] B. Fahimi, A. Kwasinski, A. Davoudi, R. S. Balog and M. Kiani, "Charge It- Powering a More Electrified Planet," in IEEE Power and Energy Magazine (IEEE EPS), vol. 9, no. 4, pp. 5464, July-Aug. 2011, doi: 10.1109/MPE.2011.941321.

[8] C. W. Gellings: "Power to the People," in IEEE Power and Energy Magazine (IEEE EPS), Vol. september/otober 2011, DOI: 10.1109/MPE.2011.941830.

[9] S. Kumar, S. Islam, and A. Jolfaei. Microgrid communications - protocols and standards. Chapter in book "Variability, Scalability and Stability of Microgrids" (pp.291326), July 2019, DOI: 10.1049/PBPO139E_ch9.

[10] I. Serban, S. Céspedes, C. Marinescu, C. A. Azurdia-Meza, J. S. Gómez and D. S. Hueichapan, "Communication Requirements in Microgrids: A Practical Survey," in IEEE Access, vol. 8, pp. 47694-47712, 2020, doi: 10.1109/ACCESS.2020.2977928.

[11] InteliLIGHT ${ }^{\circledR}$ Streetlight Management System: "inteliLIGHT ${ }^{\circledR}$ StreetLight Control Solution-- communication technologies", https://intelilight.eu/ (last retrieved on 22.06.2021).

[12] Sharda: "An Insight into the Automated Guided Vehicle (AGV) Used in the Maritime Industry", on Marine Insight, November 2019.2 Online: https://www.marineinsight.com/guidelines/an-insight-into-theautomated-guided-vehicle-agv-used-in-the-maritime-industry/ (last retrieved: 22.06.2021). 